\title{
Tiltable rotating liquid mirrors: a progress report
}

\author{
G. Gagné ${ }^{1,2}$, E. F. Borra ${ }^{1,2}$, and A. M. Ritcey ${ }^{1,3}$ \\ ${ }^{1}$ Centre d'Optique, Photonique et Lasers, Université Laval, Québec, Qc, G1K 7P4, Canada \\ e-mail: guillaume.gagne.2@ulaval.ca; borra@phy.ulaval.ca; Anna.Ritcey@chm.ulaval.ca \\ 2 Département de Physique, Génie Physique et Optique, Université Laval, Québec, Qc, G1K 7P4, Canada \\ 3 Département de Chimie, Université Laval, Québec, Qc, G1K 7P4, Canada
}

Received 20 September 2007 / Accepted 4 December 2007

\begin{abstract}
Context. We give a progress report on tiltable, nanoengineered, rotating liquid mirrors, which were discussed in previous papers. Aims. We want to develop the technology, improve reflectivities and user-friendliness. The ultimate goal is to demonstrate high-quality liquid mirrors that can be tilted by a few tens of degrees.

Methods. We coated hydrophilic liquid substrates that have poor reflectivities with a reflective layer of self-assembling metallic nanoparticles. We analyzed the wavefronts of 1-m diameter mirrors with Ronchi tests, knife-edge tests and point-spread functions (PSFs).

Results. There is significant improvement over previous work where the reflecting layer was deposited on hydrophobic oils. While previous work only demonstrated tilted low-reflectivity mirrors, we now test a high-reflectivity 1-m diameter liquid mirror tilted by 45 arcmin.

Conclusions. It is considerably easier to coat hydrophilic liquids than hydrophobic ones. We have reached a significant milestone by demonstrating a tilted, highly reflective, liquid mirror. Although this is still an immature technology, it is near the stage where it could be used in astronomy. The remaining technical challenges, for which we propose solutions, are not fundamental and could be overcome with additional work. This will be a worthwhile undertaking, considering the very low cost of liquid mirrors.
\end{abstract}

Key words. telescopes - instrumentation: miscellaneous

\section{Introduction}

Astronomical telescopes are expensive instruments. The unfortunate consequence of this is that few are built and available telescope time is thus limited. Consequently, only a few privileged individuals or teams obtain telescope time and even this elite are handicapped because they cannot obtain adequate access. A more unfortunate consequence is that data is so precious that marginal observations are sometimes analyzed and interpreted, resulting in the waste of the most precious time of all: human time. Another consequence is that research programs are often conservative, because of the risk factor inherent in original research, while it is well-established that reward is highly correlated with risk in human activities.

The cost problem is illustrated by the new generation of optical telescopes presently planned in the $30-\mathrm{m}$ to $100-\mathrm{m}$ diameter range. Their estimated costs are of the order of US\$ 1 billion each, clearly approaching the price limit that society is willing to invest for a scientific instrument. Classical astronomical telescopes are costly in large part because the primary mirrors are made of heavy glass slabs that are expensive to polish and support. The fact that heavy primary mirrors and the structures holding them must be tilted to access and track different regions of the sky further increases the final costs. It is therefore worthwhile to find new technologies that enable the fabrication of inexpensive low-weight large mirrors. One may argue that the cost of a modern classical observatory is not dominated by the cost of the mirror. This is certainly true nowadays. However, the cost of the frame itself (e.g. the bearings) will also decrease with lighter liquid mirrors and their simpler support systems. Instrumentation is a very significant cost component. However, this is partly a consequence of the fact that the telescope is expensive, hence multiple instruments are available to the telescope. These instruments are complicated and expensive because they must be rapidly interchangeable and must be highly efficient, considering the cost of telescope time. If instead telescopes were inexpensive, one could afford multiple telescopes, each one having its dedicated instrument, resulting in lower cost instrumentation. This speculative scenario has the advantage that more telescope time would be available.

Liquid mirrors offer just such a new technology. Their key advantage is that the surface of a liquid is very smooth and takes the shape of an equipotential surface, naturally generating an optical quality surface. The fact that the surface of a liquid rotating in a gravitational field takes the shape of a parabola has been used to make inexpensive mirrors having excellent surface qualities. The technology is young but its performance is well documented by laboratory tests (Borra et al. 1992; Girard \& Borra 1997; Tremblay \& Borra 2000) as well as by observations (Sica et al. 1995; Hickson \& Mulrooney 1998; Cabanac et al. 1998; Hickson \& Racine 2007).

Tiltable liquid mirrors were proposed by Borra et al. (1999) who, however, only demonstrated tiltable mirrors that had low reflectivities. This new generation of liquid mirrors has the additional important advantage over glass mirrors that they are very light. Assuming a liquid substrate having a thickness of a millimeter, which should be easy to make, and a density of one, the liquid for a 10-m diameter mirror would have a mass of only $79 \mathrm{~kg}$. Consequently, it would need a much lighter and less expensive mechanical structure than a glass mirror. In the present 


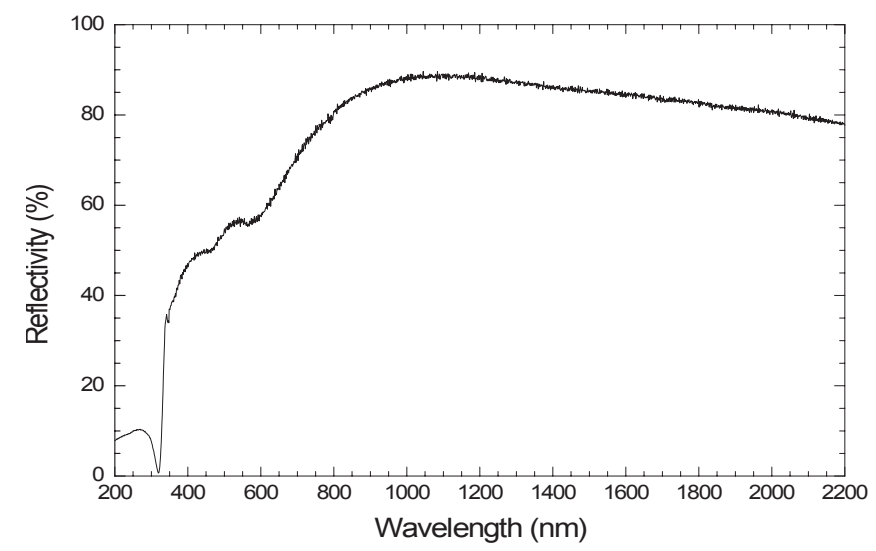

Fig. 1. Reflectivity curve of a MELLF.

progress report we demonstrate tiltable liquid mirrors having high reflectivities.

\section{Nanoengineered liquid mirrors}

The mirrors discussed in this paper are made of a thin reflective layer of silver nanoparticles supported by a liquid substrate. Stable interfacial assemblies of silver nano-particles have been previously reported in the literature and are known as MEtal Liquid-Like Films, or MELLFs (Yogev \& Efrima 1988; Gordon et al. 1989). MELLFs were originally used in spectroscopic experiments to study the chemicals that coated the colloidal grains.

The preparation of a MELLF involves the fabrication of silver nanoparticles coated with an organic protective layer. Experimental details have been previously described (Gingras et al. 2006). Our early MELLFs were spread on water. Borra et al. (2004) reported on a major improvement of the technology: the successful subsequent spreading of the MELLF at the surface of an organic oil. This feat greatly extends the range of physical and chemical characteristics of the available liquid substrates and also solves a major problem with water-based liquids: namely, evaporation. However, reflectivities on oil were lower than those found for water-based MELLFs and it was difficult to coat large mirrors. At the time, we were optimistic that we would rapidly solve these problems but the task has turned out to be much harder than we expected. More recently, we have turned our attention to a new approach based on the use of viscous hydrophilic liquids rather than organic oils. The inherently high surface energy of hydrophilic liquids thermodynamically favors the spreading of thin films at the air-liquid interface. This means that the use of surfactants, required for the spreading of the MELLF on oil, can be eliminated.

Earlier in the project, MELLFs were made by hand and the process was very labor intensive, requiring more than one hundred hours to make enough coated particles to cover a $1-\mathrm{m} \mathrm{di-}$ ameter mirror. To cut down on labor we made a small simple motorized, computer-controlled MELLF machine. It now takes $30 \mathrm{~h}$ to make enough MELLF for a 1-m mirror. The process is semi-automated and only requires about $8 \mathrm{~h}$ of supervision. A more sophisticated machine would certainly further reduce this time. The quality of the MELLF made by the machine is comparable to the quality of handmade MELLFs. Figure 1 shows a recent reflectivity curve of a MELLF spread on water.

The main advantage of working with hydrophilic liquids is that coating is far easier. We no longer depend on surfactants to bring the MELLF to the surface of the oil as in Borra et al. (2004) but simply spray the MELLF on the surface of the hydrophilic liquid. Unfortunately this technique is limited to liquids with relatively high surface tensions (greater than $40 \mathrm{mN} \mathrm{m}^{-1}$ ), greatly restricting the number of liquids that can be used.

\section{Optical tests}

\subsection{Tests on small flat mirrors}

To develop the basic technology, we experimented first with small flat mirrors since they are easier to handle and test. We tested them with a Fizeau interferometer, working at a wavelength of 632.8-nm. We restricted ourselves to hydrophilic liquids, including some low molecular weight polymers, that are commercially available and are inexpensive (Table 1).

Kelkogel $\mathrm{F}$ is a polysaccharide sold as a powder by the company CP Kelco. It can be mixed with ethylene glycol to make liquids of varying viscosities. While we found that MELLFs spread well on Kelkogel, the reflectivity is poor, rendering it useless for astronomical applications. We did not experiment extensively with Kelkogel. Poly(ethylene glycol-ran-propylene glycol) is a copolymer of ethylene glycol and of propylene glycol. It gave excellent surfaces and initial reflectivities that unfortunately deteriorated to unacceptable levels within a few hours. Because of the poor reflectivities obtained with some of the liquids, we restricted our interferometric tests to the subset marked with an asterisk in Table 1. Our best results were obtained with ethylene glycol and glycerol. Since both liquids had the highest surface tensions, our tests suggest that only high surface tension liquids can be coated.

Although hydrophilic liquids facilitate spreading of the surface film of nanoparticles, they are not without problems. For example, the surface qualities of the uncoated hydrophilic liquids are typically worse than the surfaces of liquid metals or hydrophobic liquids such as silicon oil. Although we have not investigated why this is the case, a logical explanation may come from the fact that the liquids, being hydrophilic, absorb water from the air. Presumably, during the pouring process, inhomogeneities are created by regions having slightly different water contents and therefore different densities. It only takes small density inhomogeneities for buoyancy to introduce defects of the order of tens of nanometers. A second drawback noted with respect to the hydrophobic liquids is that it takes longer for the surface to stabilize after the liquid has been poured. Finally, the viscosity of a hydrophilic liquid exposed to ambient air decreases markedly with time due to the uptake of water. We find that it takes only $24 \mathrm{~h}$ to reduce the viscosity of glycerol from $685 \mathrm{cSt}$ to $200 \mathrm{cSt}$ at a $65 \%$ ambient humidity. This is not a major problem because water absorption can be controlled relatively easily. For our tilt experiments, a dehumidifier was therefore placed in the tent that surrounds the mirror and the testing tower. A mylar cover was also placed on top of the mirror to limit exposure to air.

Simply pouring the MELLF on hydrophilic liquids gives unacceptable surface quality, yielding surfaces that are covered with bubbles and spots. We experimented with several coating techniques, and found that spraying the MELLF on the surface of the liquid gives the best results. Spraying is carried out with a commercial spray-gun of the type used to paint automobiles. One must experiment with spraying (e.g. MELLF concentration, pressure, etc.) to obtain the best results. For example, spraying must be done with a single continuous movement, otherwise dried patches overlap.

We experimented with two different types of MELLFs. Firstly, we experimented with classical MELLFs that only 
Table 1. List of the hydrophilic liquids that we tested. The asterisks marks the liquids that gave reasonable reflectivities and were subsequently tested interferometrically.

\begin{tabular}{lcccc}
\hline \hline Liquids & $\begin{array}{c}\text { Surface tension } \\
(\mathrm{mN} / \mathrm{m})\end{array}$ & $\begin{array}{c}\text { Viscosity (temperature) } \\
(\mathrm{cSt})\end{array}$ & Molecular weight $\left(m_{\mathrm{n}}\right)$ & Composition \\
\hline Poly(EG-ran-PG)* & 40.2 & $650\left(25^{\circ} \mathrm{C}\right)$ & 2500 & EG, 75wt $\%$ \\
Poly(EG-ran-PG) & $(\ldots)$ & $35000\left(25^{\circ} \mathrm{C}\right)$ & 12000 & EG, $75 \mathrm{wt} \%$ \\
Poly(EG-ran-PG) monobutyl ether & 36.8 & $3600\left(20^{\circ} \mathrm{C}\right)$ & 3900 & EG, 50wt $\%$ \\
Glycerol* & 64.0 & $685\left(25^{\circ} \mathrm{C}\right)$ & $(\ldots)$ & $(\ldots)$ \\
Poly(PG) & $(\ldots)$ & $150\left(25^{\circ} \mathrm{C}\right)$ & 1000 & $(\ldots)$ \\
PPG-PEG-PPG & 51.0 & $480\left(77^{\circ} \mathrm{C}\right)$ & 2000 & PEG, 50wt $\%$ \\
PEG-PPG-PEG & 34.4 & $850\left(25^{\circ} \mathrm{C}\right)$ & 2900 & PEG, $40 \mathrm{wt} \%$ \\
Poly(dimethylsiloxane) & 21.1 & $500\left(25^{\circ} \mathrm{C}\right)$ & $(\ldots)$ & $(\ldots)$ \\
Poly(EG) & 43.5 & $4.3\left(100^{\circ} \mathrm{C}\right)$ & 200 & $(\ldots)$ \\
Ethylene glycol* & 48.0 & $20\left(25^{\circ} \mathrm{C}\right)$ & $(\ldots)$ & $(\ldots)$ \\
\hline
\end{tabular}
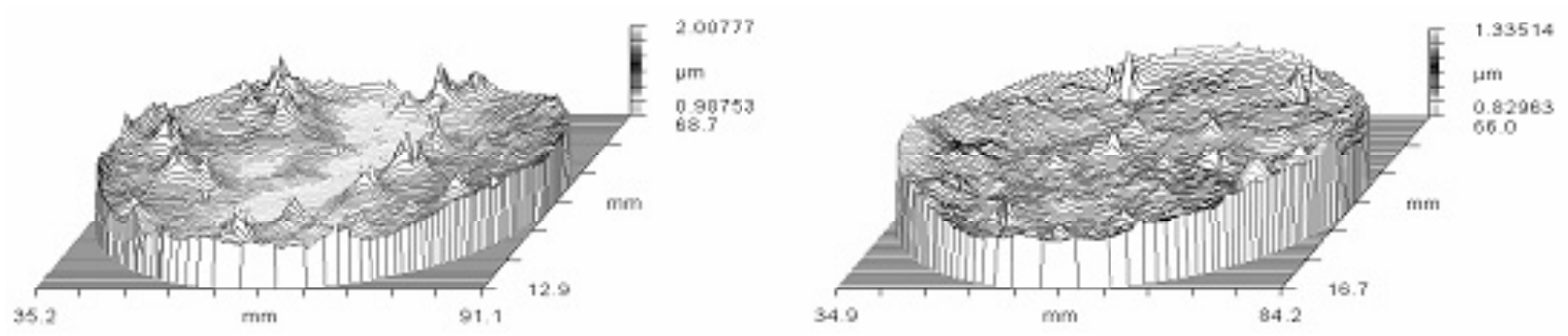

Fig. 2. Wavefronts of small flat mirrors obtained for glycerol coated with the water MELLF (left) and with the ethylene glycol MELLF (right). The wavefront of the water MELLF has an $\mathrm{rms}=\lambda / 6$ while the ethylene glycol MELLF has an $\mathrm{rms}=\lambda / 11$.

contained the residual water that is part of the process. Secondly, we experimented with MELLFs mixed in equal proportions with ethylene glycol. Figure 2 shows the wavefronts obtained for glycerol coated with the classical MELLF and with the MELLF diluted with ethylene glycol. The spikes seen in theses wavefronts are responsible for the observation that the MELLF pointspread function (PSF) is wider than the mercury PSF between 0.4 and 1.2 arcsec (see Fig. 4). The ethylene glycol MELLF gave a better surface quality.

The results obtained over different mirrors vary, probably due to fact that we spray by hand. Surface quality can be further improved by additional spraying with 1,2-dichloroethane, a technique discussed in the next section.

\subsection{Ronchi tests of rotating mirrors (MELLF on ethylene glycol)}

We analyzed ethylene glycol supported 1-m mirrors with Ronchi tests in the small testing tower described in Girard \& Borra (1997). We did not carry out interferometric tests on large mirrors because they are best suited for detecting defects on large scales, while our liquid mirrors have small-scale defects. On the other hand, Ronchi tests excel at detecting small-scale defects. Ronchi tests measure slopes and are very sensitive to slope errors. They are capable of detecting defects smaller than 1/100 wave. The bars of our Ronchi ruling subtend $12 \operatorname{arcsec}$ so that slope deviations substantially less than 1 arcsec can easily be seen. We did not use null lenses. Consequently, our Ronchigrams show the spherically aberrated signatures of parabolas tested at their centers of curvature (Cornejo-Rodriguez 1978).

Our tilt experiments (Sect. 4) were only done with glycerol because it was the only liquid, among those that we tested, which had reasonably high viscosity and gave satisfactory reflectivity and surface quality. The data discussed below were obtained on a 1-m diameter mirror having a layer of ethylene glycol 3-mm
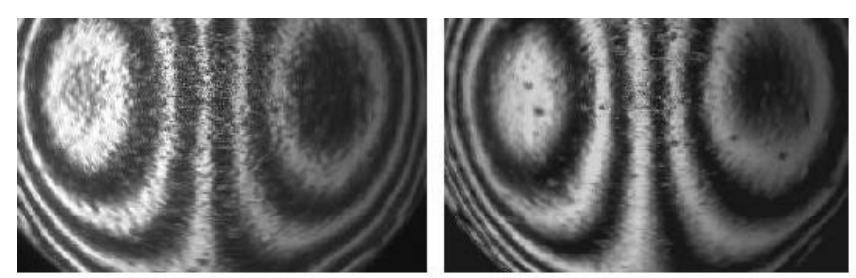

Fig. 3. Ronchigrams of 3-mm ethylene glycol coated with MELLF before (left) and after (right) spraying with dichloroethane. The smallscale defects visible in the eyes of the Ronchigrams have lower amplitudes. The more substantial improvement to the higher frequency defects cannot be seen because of the limited resolution of the CCD detector. The improvement at higher frequencies can readily be seen in the wings of the PSFs (Figs. 4 and 5).

thick. Figure 3 shows a Ronchigram of the 1-m ethylene glycol mirror after coating it with MELLFs. The surface is marred with many small-scale defects. To improve it, we then sprayed the surface again with pure dichloroethane. Although there remain defects, Fig. 3 shows a noticeable improvement. We experimented with several 1-m mirrors, finding a similar pattern of improvement after repeated spraying with pure dichloroethane. Sometimes we had to spray the mirrors several times to find a reasonable improvement.

\subsection{Point spread function (MELLF on ethylene glycol)}

The point spread function is given by the Fourier transform of the wavefront. It therefore gives quantitative information on surface defects at all spatial frequencies, including very high frequencies. It complements the Ronchi and knife-edge tests, which are qualitative but visually display the defects. We used the same testing tower and equipment described in Tremblay \& Borra (2000). Over the years, the testing equipment has degraded 


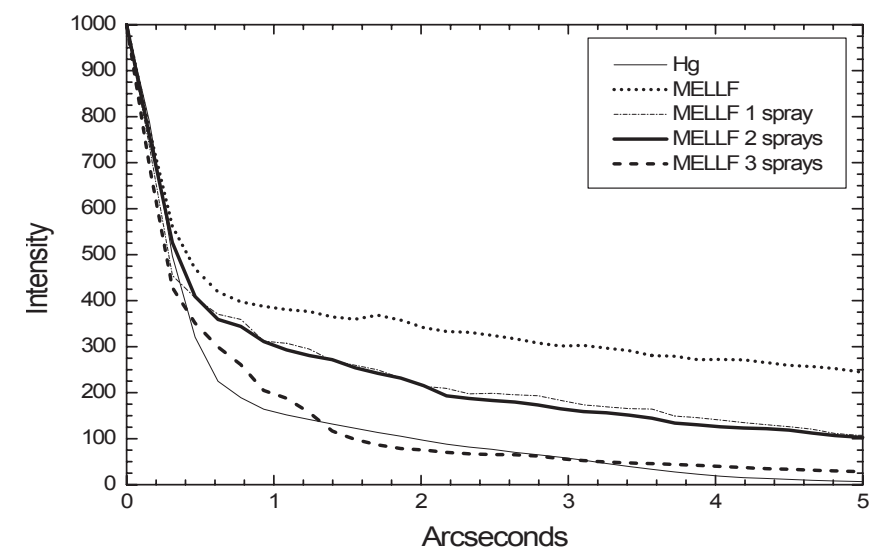

Fig. 4. The central region of the PSF of a mercury mirror is compared to the PSF of a MELLF mirror, to the MELLF mirror with a single spray of dichloroethane and then after two additional sprays. They are $1-\mathrm{m}$ diameter rotating mirrors.

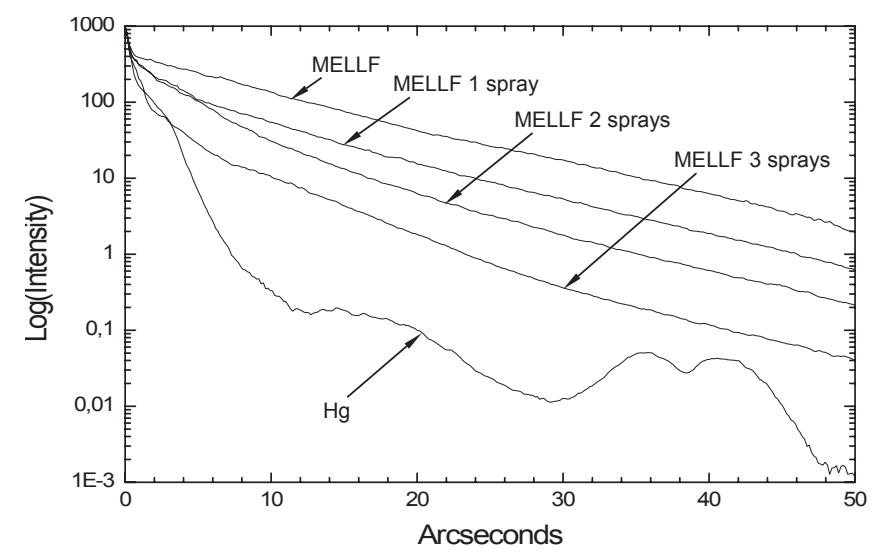

Fig. 5. The PSF of a mercury mirror is compared to the PSF of a MELLF mirror, to the MELLF mirror with a single spray of dichloroethane and then after two additional sprays. They are 1-m diameter rotating mirrors.

significantly, giving more scattered light. Seeing in the testing tower, which was outstanding, has also deteriorated to the typical seeing measured in testing towers. Consequently, the quality of the MELLF mirror is best quantified by comparing it to the PSF of a mercury mirror analyzed in a similar setup. We only tested horizontal mirrors because testing inclined ones would have necessitated a prohibitively complex testing system. We find that the PSF of the MELLF-coated mirror is considerably worse than the PSF of the mercury mirror; however there is considerable improvement after spraying the MELLF mirror with dichloroethane. Figures 4 and 5 compare the PSFs of the same MELLF mirror before and after a single spray of dichloroethane, and then again after two additional sprays of dichloroethane. We can see a noticeable improvement. The PSF of the original MELLF mirror had a FWHM of 0.82 arcsec that decreases to 0.58 arcsec after a single spray, and then to 0.54 arcsec after 2 additional sprays. Figure 5 shows scattered light dramatically improved after 3 sprays, such that PSF is only slightly higher than for the mercury mirror. The data thus confirms the conclusions reached in the previous section. Figure 6 can be used to quantitatively evaluate the improvements with spraying. It shows how selected representative regions of the PSF improve with spraying. The PSFs are normalized with respect to the PSF of a mercury mirror.

\section{Tilted liquid mirrors}

We did not carry out interferometric tests because building a facility capable of interferometrically testing tilted rotating mirrors would have been prohibitively difficult. Furthermore, while interferometry excels at quantitatively detecting defects on relatively large scales, tilted liquid mirrors have small-scale defects (Borra et al. 1999). Knife-edge and Ronchi tests excel at detecting small scale defects. Because the Ronchi test is qualitative and because we are only interested in tilt effects, we qualitatively compare the Ronchigrams of the tilted mirrors to the Ronchigrams of the horizontal mirrors.

We could not obtain layers of glycerol thinner than $3.5-\mathrm{mm}$, presumably because it has relatively high surface tension. One should be able to circumvent this limit by carving a ditch around the mirror, like we do for mercury mirrors (Borra et al. 1992). Pumpdown is probably a poor solution for a high-viscosity liquid. Spraying is probably a better way to proceed. Rendering the surface wettable is another option (e.g. by spincasting with a wettable material). Figure 7 shows Ronchigrams of the 1-m mirror for various tilt angles.

As the tilt angle increases, the large-scale appearances of the Ronchigrams are similar to those of the untilted mirror, indicating that, over distances of the order of $10-\mathrm{cm}$, there is little change with tilting. The surface returns to its unperturbed state within one minute of time after it is tilted back to zero arcminutes. Note that the observation that the shapes of the Ronchigrams are not perfectly identical is simply due to the position of the Ronchi ruling on the caustic having changed (e.g. Fig. 9.9 of Cornejo-Rodriguez 1978). Note also that the Ronchi test is a qualitative test and Fig. 7 does not prove that the surface remains perfectly parabolical as it is tilted. It only shows that it remains close to the shape of a parabola. However, we are confident, on the basis of our experience with liquid mirrors that the liquid closely follows a parabola over distances greater than a few centimeters. As we increase the tilt angle, an increasing number of speckles appear on the Ronchigrams; the same effects were reported by Borra et al. (1999) for uncoated silicon oil mirrors. Their lateral dimensions do not change noticeably with tilt angle. They are fixed on the surface and rotate with the mirror. Borra et al. (1999) could only speculate on the origin of these speckles. Subsequent experiments discussed in Dallaire et al. (2004) show that the speckles are caused by printthrough. Printthrough, first reported on mercury liquid mirrors (Borra et al. 1992), is caused by the movement of the liquid over small defects present on the solid surface of the container that holds the liquid. For tilted liquid mirrors the movement is due to the fact that the liquid is subject to a force having the period of rotation of the mirror and amplitude $g \sin (\theta)$, where $g$ is the gravitational acceleration and $\theta$ the tilt angle. Dallaire et al. (2004) discuss printthrough in detail and estimate the attenuation factors (of the order of a few 100) between the amplitudes at the solid and liquid surface. Consequently, one would need a container having roughness of less than $0.1 \mathrm{~mm}$. The spiral-like defect seen in Fig. 7 for the horizontal mirror is due to a problem during stabilization for this particular mirror. The spiral for all the remaining inclinations (which increases with inclination) is due to printthrough of a spiral defect at the center of the solid surface of the container. It is caused by Coriolis forces on the liquid polyurethane poured in the container during spincasting. The surface of the container is spincast to give it a parabolic shape (Borra et al. 1992). The last image in Fig. 7 shows the spiral defect on the solid surface of the container. The deviations on the solid surface have an rms value of 75 microns. 

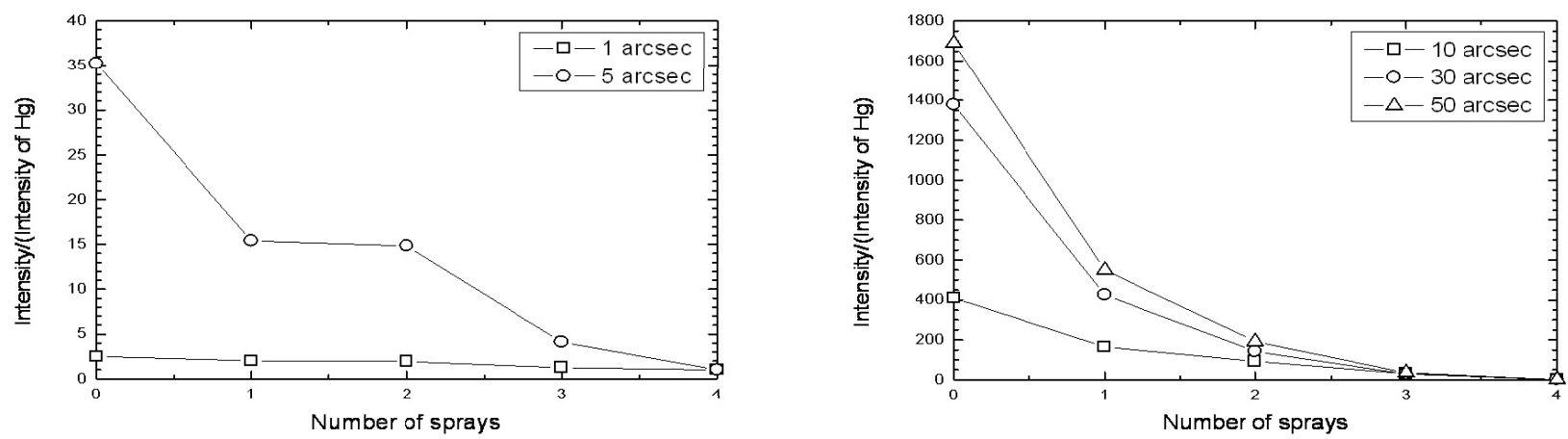

Fig. 6. This figure shows the improvement with spraying at selected locations of the PSF and compares it to the PSF of mercury. The PSFs are normalized with respect to the PSF of a mercury mirror. The horizontal axis gives the number of sprays; however, the fourth spray is actually the value for mercury which is 1.00 , because of the normalization for all locations.

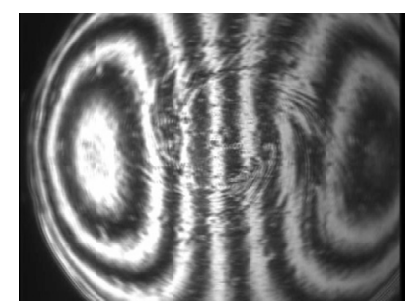

(a) 0 arc minutes

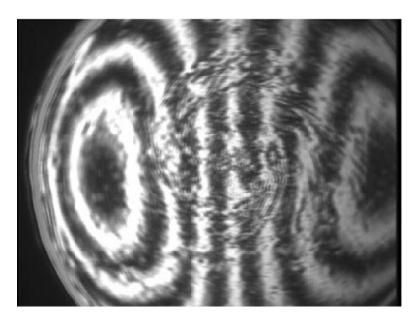

(c) 15 arc minutes

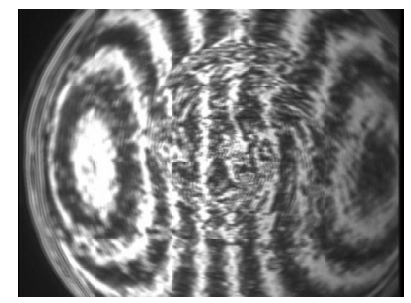

(e) 30 arc minutes

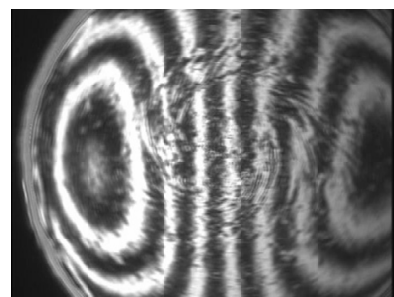

(b) 10 arc minutes

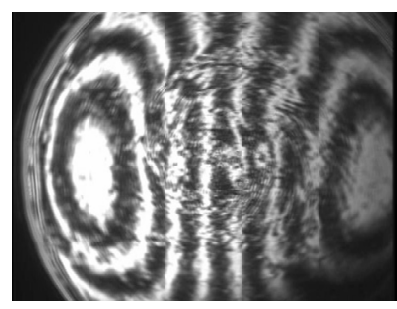

(d) 20 arc minutes

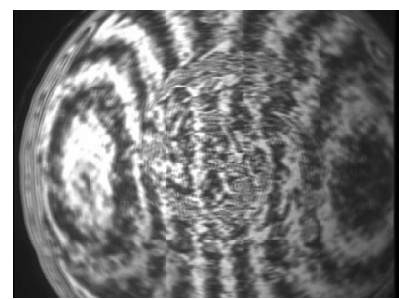

(f) 45 arc minutes

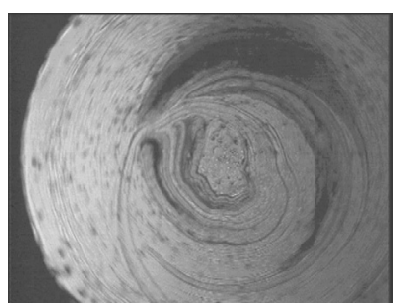

(g) Container

Fig. 7. Ronchigrams of the 1-m mirror at various tilt angles. The surface returns to its unperturbed state within a minute of time after it is tilted back to zero arcminutes. Defects on the solid surface of the container print through to the surface and are attenuated by a factor of about 100 . The last image in this figure shows the spiral defect on the solid surface of the container that prominently prints through to the liquid surfaces. Its rms amplitude on the solid top surface of the container is 75 microns and therefore of the order of 1 micron on the liquid surface. We understand printthrough and know how to minimize it (see text).

Dallaire et al. (2004) discuss at length how to minimize printthrough. This can be done by increasing the viscosity of the liquid and decreasing the thickness of the liquid layer. Printthrough is slightly decreased by the MELLF.

\section{Conclusion}

This article gives a progress report on nanoengineered rotating liquid mirrors. We coated hydrophilic substrates with reflective layers of self-assembling metallic nanoparticles called MELLFs. There is significant improvement over previous work where the reflecting layer was deposited on hydrophobic oils (Borra et al. 2004). We demonstrate for the first time a tilted liquid mirror that has a high-reflectivity, since Borra et al. (1999) only demonstrated a low-reflectivity tilted mirror.

The fact that hydrophilic liquids absorb water also causes problems, because water absorption significantly decreases their viscosities. For our tilt experiments, we had to put a dehumidifier in the tent that surrounds the mirror and the testing tower, as well as a mylar cover on top of the mirror. We also found that the surface qualities of the uncoated hydrophilic liquids are worse than the surfaces of liquid metals or hydrophobic liquids such as silicon oil. This is presumably caused by buoyancy effects, due to density inhomogeneities caused by water absorption.

We restricted our tests to commercially available hydrophilic liquids. Only three were successfully coated: ethylene glycol, glycerol and poly(EG-ran-PG). However, the reflectivity of mirrors spread on poly(EG-ran-PG) deteriorated after a few hours, rendering it useless. The liquids successfully coated were those that had the highest surface tensions. The way the MELLF is spread on hydrophilic liquids is very important. After experimenting with coating techniques, we find that spraying the MELLF on the surface of the liquid with a commercial spraygun gives the best results. We experimented with a MELLF mixed with ethylene glycol, finding that it gives better results than classical MELLFs mixed with water.

We analyzed 1-m mirrors with Ronchi tests, finding that surface qualities are not as good as those of mercury mirrors. The surfaces are marred by numerous small-scale defects. We improved them considerably by spraying them again with pure dichloroethane; however, defects, essentially due to the amalgams of silver nanoparticles, remained. We also analyzed PSF profiles. Because the PSF is given by the Fourier transform of the wavefront, it gives quantitative information on surface defects at all spatial frequencies and complements the Ronchi tests. Our PSF measurements confirm that spraying with pure dichloroethane noticeably improves surface quality. In 
particular, scattered light is dramatically reduced after 3 sprays and is only slightly worse than what we obtain for a mercury mirror. Figure 6 shows the improvement with spraying at selected locations of the PSF.

We carried out Ronchi tests on tilted glycerol mirrors. We only experimented with glycerol since it was the only viscous liquid, among those tested, that gave good surface quality and reflectivity on tests carried out with small flat mirrors. As we increase the tilt angle, we see the same effects reported by Borra et al. (1999) for uncoated silicon oil mirrors. The surfaces are marred by an increasing number of speckles caused by printthrough of the defects on the surface of the container (Dallaire et al. 2004). Printthrough can be significantly reduced by using very viscous liquids and thin liquid layers (Dallaire et al. 2004).

MELLF-coated liquid mirrors have the additional advantage that viscous liquids are less sensitive to vibrations and wind induced perturbances than liquid metals. This is an important consideration because the wind caused by the rotation of the mirror induces spiral-like surface defects (Borra et al. 1992; Hickson $\&$ Racine 2007) that impose limits on the diameter of liquid mirrors.

Although we have demonstrated a major improvement in user-friendliness and performance, this is still an immature technology and there is work to be done before it reaches the performance and robustness expected for routine astronomical applications. There are several improvements to be made. Firstly, we must find a higher viscosity (about $5000 \mathrm{cSt}$ ) hydrophilic liquid that will allow us to tilt the mirror to at least 10 degrees. Ionic liquids are excellent candidates since many of them are hydrophilic and there are at least $10^{6}$ simple ionic liquids, and $10^{18}$ ternary ionic liquid systems, giving a phenomenal choice for optimizing the properties of the liquid substrate. In addition, we must decrease printthrough to tolerable levels. These are only technical improvements. On the other hand, although we must still improve surface quality, a mirror having the quality shown by the mirrors with 3 sprays in Figs. 4 and 5 is already adequate for some less demanding astronomical applications and applications in some fields of science (e.g. atmospheric science; Sica et al. 1995). It will be worthwhile making the effort because, based on our experience so far, tiltable liquid mirrors promise to be very inexpensive and easy to make, ushering in an era of inexpensive telescopes and readily available telescope time.

Acknowledgements. This research was supported by the Natural Sciences and Engineering Research Council of Canada.

\section{References}

Borra, E. F., Content, R., Girard, L., et al. 1992, ApJ, 393, 829

Borra, E. F., Ritcey, A., \& Artigau, E. 1999, ApJ, 516, L115

Borra, E. F., Ritcey, A. M., Bergamasco, R., et al. 2004, A\&A, 419, 777

Brousseau, D., Borra, E. F., Ruel, H.-J., \& Parent, J. 2006, Optics Express, 14, 11486

Cabanac, R., Borra, E. F., \& Beauchemin, M. 1998, ApJ, 509, 309

Cornejo-Rodriguez, A. 1978, in Optical Shop Testing, ed. D. Malacara (New York: Wiley)

Dallaire, M., Bergamasco, R., \& Borra, E. F. 2004, Exp. Astron., 16, 85 Gingras, J., Dery, J.-P., Yockell-Lelievre, H., et al. 2006, Colloids and Surfaces A: Physicochem. Eng. Aspects, 279(1-3), 79

Girard, L., \& Borra, E. F. 1997, Appl. Opt., 36, 25, 6278

Gordon, K. C., McGarvey, J. J., \& Taylor, K. P. 1989, J. Phys. Chem., 93, 6814

Hickson, P., \& Mulrooney, M. 1998, ApJS, 115, 35

Hickson, P., \& Racine, R. 2007, PASP, 119, 456

Sica, R. J., Sargoytchev, S., Borra, E. F., et al. 1995, Appl. Opt., 34, 6925

Tremblay, G., \& Borra, E. F. 2000, Appl. Opt., 39, 5651

Yogev, D., \& Efrima, S. 1988, J. Phys. Chem., 92, 5754 